\title{
Quantitative analysis of myocardial fibrosis in normals, hypertensive hearts, and hypertrophic cardiomyopathy
}

\author{
MASARU TANAKA, HISAYOSHI FUJIWARA, TOMOYA ONODERA, \\ DER-JINN WU, YOSHIHIRO HAMASHIMA, CHUICHI KAWAI \\ From the Third Division, Departments of Internal Medicine and Pathology, Kyoto University, Sakyo-ku, \\ Kyoto, fapan
}

SUMMARY The distribution of fibrosis was studied quantitatively in the entire left ventricular wall of a transverse slice of the heart from 10 necropsy cases of hypertrophic cardiomyopathy, 10 cases of hypertensive heart disease, and 20 normal adults. The percentage area (mean (SD)) of fibrosis in the left ventricular wall in hypertrophic cardiomyopathy $(10.5(4.3) \%)$ was significantly greater than that in hypertensive heart disease $(2.6(1.5) \%)$ or in normal hearts $(1 \cdot 1(0.5) \%)$. In hypertrophic cardiomyopathy the percentage area of fibrosis was greater $(13 \cdot 1$ $(4.8) \%$ ) in the ventricular septum than in the left ventricular free wall $(7 \cdot 7(4 \cdot 2) \%)$ whereas in hypertensive heart disease and normal hearts values in these two areas were similar. The percentage area of fibrosis in the left ventricular free wall (where myocardial fibre disarray was not extensive even in hypertrophic cardiomyopathy) was greater in hypertrophic cardiomyopathy than in hypertensive heart disease. The percentage area of fibrosis correlated with heart weight in hypertensive heart disease, but not in hypertrophic cardiomyopathy. These results suggest that widespread fibrosis in hypertrophic cardiomyopathy cannot be explained by cardiac hypertrophy alone, and that disarray and other factors are also important in pathogenesis. The increase in the percentage area of fibrosis from the outer to the inner third of the left ventricular free wall in hypertrophic cardiomyopathy and in hypertension probably reflected transmural gradients of wall stress and myocardial fibre diameter.

Although fibrosis is not specific to hypertrophic cardiomyopathy, its quantification and analysis of its regional distribution provide information that is useful in investigating the pathophysiology of the disorder.

Although myocardial fibrosis is an important finding in hypertrophic cardiomyopathy, ${ }^{12}$ most studies have concentrated on myocardial fibre disarray ${ }^{3-5}$ and myocytic hypertrophy. ${ }^{67}$ There are few reports on fibrosis in hypertrophic cardiomyopathy and they have a number of drawbacks. Fibrosis was assessed semi-quantitatively by a grading method ${ }^{89}$ and evaluation of fibrosis was limited to a small area of the left ventricle. ${ }^{1011}$ Hence the present study was

Requests for reprints to Professor Chuichi Kawai, The Third Division, Department of Internal Medicine, Faculty of Medicine, Kyoto University, Sakyo-Ku, Kyoto 606, Japan.

Accepted for publication 28 January 1986 undertaken to determine, for the first time in quantitative terms, the extent of myocardial fibrosis in the entire left ventricular wall of the transverse slice at the level of maximal septal hypertrophy in hypertrophic cardiomyopathy; and to compare this with the distribution of fibrosis in hypertensive heart disease or normal adult hearts.

\section{Patients and methods}

Forty hearts from necropsy cases were studied-10 with hypertrophic cardiomyopathy, 10 with concentric hypertrophy secondary to hypertension, and 20 normal adult hearts. The Table lists the necropsy 
Table Necropsy data in patients with hypertrophic cardiomyopathy, hypertensive heart disease, and in normal controls

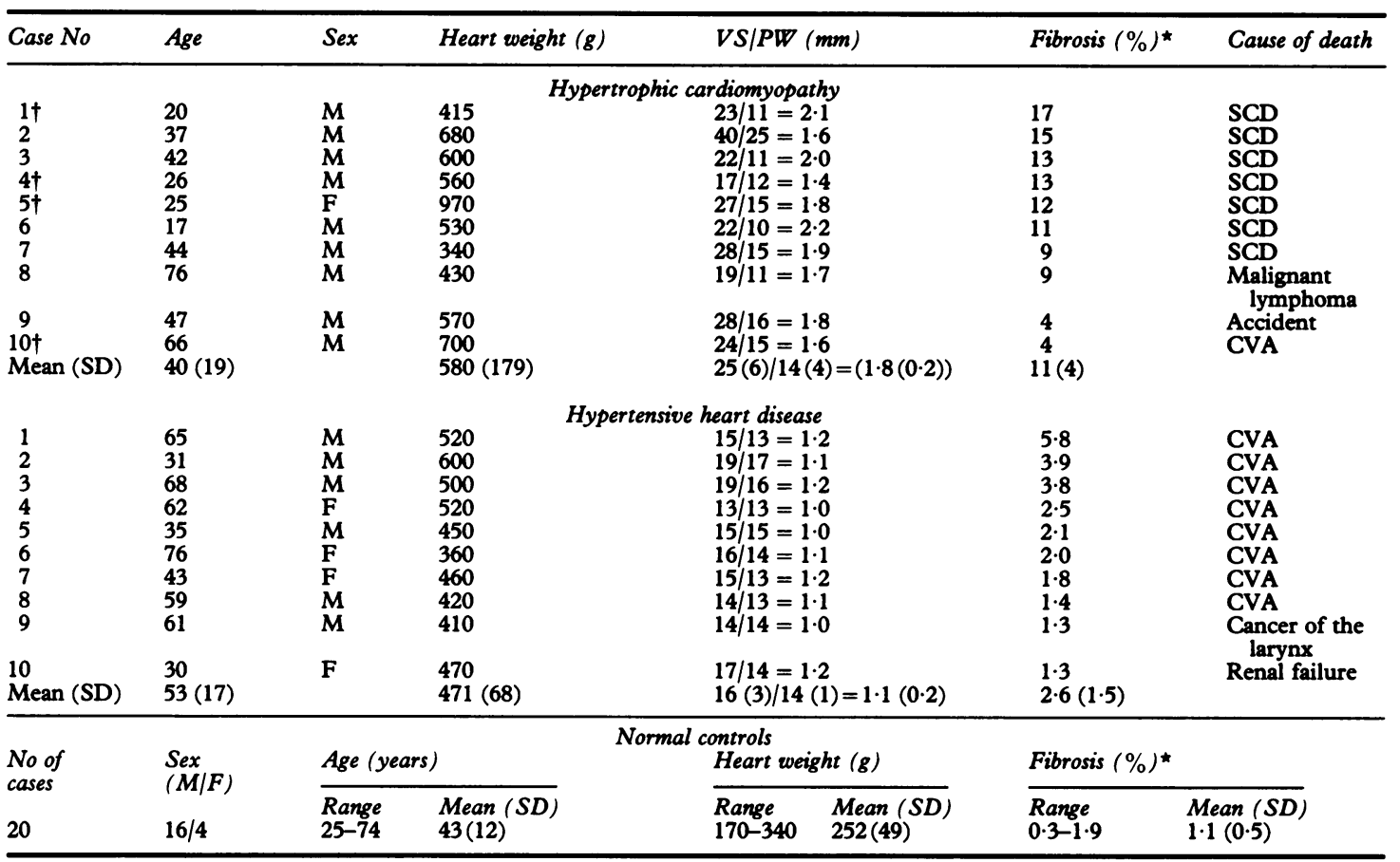

VS, ventricular septum; PW, left ventricular posterior wall; SCD, sudden cardiac death; CVA, cerebrovascular attack. $\star$ Percentage area of fibrosis in left ventricular wall. tLeft ventricular outflow tract obstruction.

data. There was no significant difference in age and sex among the three groups, nor in heart weight between cases of hypertrophic cardiomyopathy and of hypertensive heart disease.

Eight of the 10 cases of hypertrophic cardiomyopathy were diagnosed by both clinical and pathological criteria. In the remaining two cases the diagnosis was based on pathological examination. The pathological criterion for hypertrophic cardiomyopathy was the presence of asymmetric septal hypertrophy with widespread myocardial fibre disarray in the absence of hypertensive, valvar, congenital, or infectious diseases that could have produced left ventricular hypertrophy. None of the cases showed important stenosis of the coronary arteries or acute or old myocardial infarction. Sudden death from cardiac causes occurred in seven patients and the others died of extracardiac causes (malignant lymphoma, accident, and cerebral infarction). There was left ventricular outflow tract obstruction in four patients.

Hypertensive heart disease was diagnosed on the basis of left ventricular concentric hypertrophy due to long term overt hypertension $(>160 / 95 \mathrm{~mm} \mathrm{Hg}$ ) in a heart weighing $\geqslant 350 \mathrm{~g}$. Hearts with hyper- tensive disease and severe coronary arterial stenosis ( $>75 \%$ luminal narrowing) were excluded from this study. Definite congestive heart failure was not evident in any case of hypertrophic cardiomyopathy or hypertensive heart disease. All cases of hypertensive heart disease died of extracardiac causes (Table).

A heart was regarded as normal if there was no clinical or pathological evidence of heart disease; if the coronary arteries did not show $\geqslant 50 \%$ luminal narrowing; and if it weighed $\leqslant 350 \mathrm{~g}$.

\section{QUANTIFICATION OF MYOCARDIAL FIBROSIS}

The hearts were fixed in $10 \%$ formalin and cut transversely from the base to the apex serially at $1 \mathrm{~cm}$ intervals. In the hearts with hypertrophic cardiomyopathy, the slice showing maximal septal hypertrophy was used. In the normal and hypertensive hearts we studied a slice from the middle third of the heart. Each slice was divided into 6-8 blocks, which were sectioned at a thickness of $4 \mu \mathrm{m}$. These sections were stained with Masson's trichrome and haematoxylin and eosin.

The $4 \mu \mathrm{m}$ thick preparations stained with Masson's trichrome were directly enlarged $(x 10)$ by a photo enlarger and the blue areas showing fibrosis 

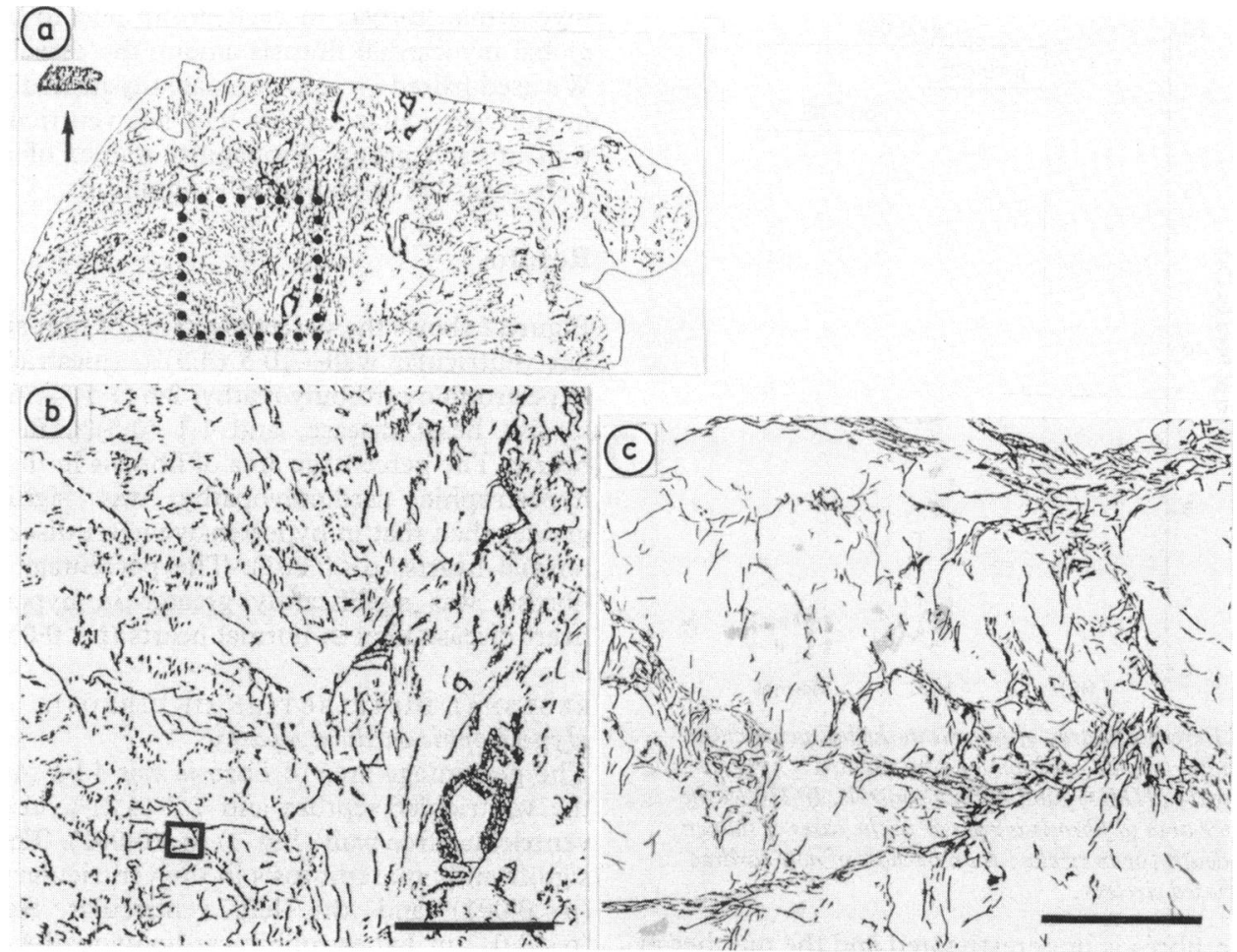

Fig. 1 Traced pictures of fine cardiac fibrosis stained blue with Masson's trichrome. (a) Fine fibrosis traced at $\times 10$ magnification. The percentage area of fibrosis of this section was $17 \%$. Actual size of tissue section is shown upper left (arrow) for comparison. (b) Original size of tracing at $\times 10$ magnification obtained from the square indicated in (a). Bar indicates $2 \mathrm{~mm}$. (c) Original size of tracing at $\times 250$ magnification obtained from the square indicated in $(b)$. Bar indicates $80 \mu m$. Note that at low magnification (b), not all of the fine collagen fibres traced at high magnification $(c)$ are detected and the percentage area of fibrosis is underestimated. At low magnification ( $b$ ), however, a small bundle of collagen fibres seen at high magnification (c) is traced as a single line and the percentage area of fibrosis is overestimated. As a result, the percentage area of fibrosis measured at $\times 10$ correlates well with that at $\times 250(y=1 \cdot 08 x-1 \cdot 1, r=0.95, p<0.01) .{ }^{12}$

were carefully traced with a fine black pen (Fig. 1) These fibrotic areas were measured semiautomatically by a multipurpose colour image processor (model VIP-21C, Olympus). This processor consisted of a colour television camera, a high resolution colour television monitor, an image processing and calculation unit with a microprocessor, a light pen controller with a microprocessor, and a list printer.

Papillary muscles were excluded from the measurement of fibrosis. Percentage area of fibrosis was calculated and used as the index of the degree of fibrosis. The percentage area of fibrosis in the entire left ventricular wall of the transverse slice was obtained by dividing the sum of the fibrotic areas of all sections by that of the total tissue areas. For detailed examination of the intramural distribution of fibrosis, the sections were subdivided into the fol- lowing four regions (anterior, lateral, posterior wall of the left ventricular free wall, and the ventricular septum) and the percentage area of fibrosis in each region was calculated separately. The percentage area of fibrosis was also calculated separately in the outer, middle, and inner third of the left ventricular free wall, and in the right, middle, and the left third of the ventricular septum.

In all cases fibrosis was traced and quantified independently by two morphologists who did not know to which patient the tissue section belonged. They often recognised the patient's disease, however, when a particularly large section with widespread myocardial fibre disarray strongly suggested the presence of hypertrophic cardiomyopathy. The variance between these observers for the percentage areas of fibrosis was $<0.8 \%$.

At a magnification of $\times 10$ the number of single 


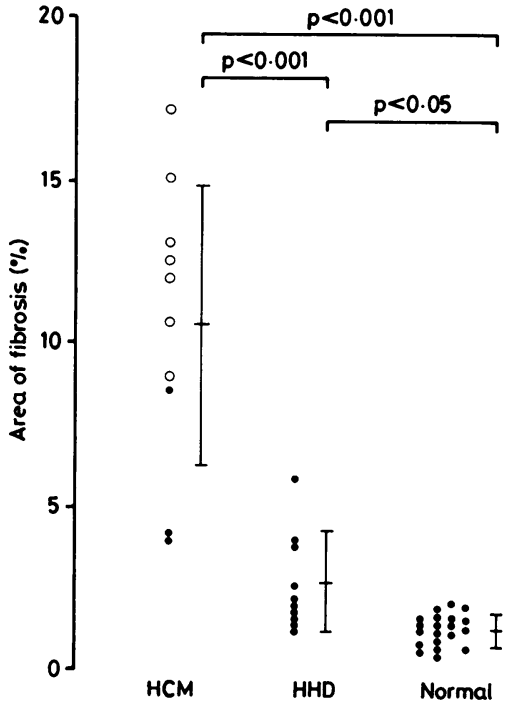

Fig. 2 Percentage area of fibrosis in the left ventricular wall in hypertrophic cardiomyopathy (HCM), hypertensive heart disease (HHD), and normal controls. In HCM the percentage area of fibrosis is greater in the cases of sudden cardiac death (open circles) than in those of non-cardiac death (closed circles).

collagen fibres is underestimated and the number of collagen fibres in small bundles is overestimated compared with results obtained at a magnification of $\times 250 .{ }^{12}$ Although individual fibres, which are detectable at $\times 250$, are not detectable at $\times 10$ the overall percentage of fibrosis calculated at both magnifications is similar (Fig. 1). ${ }^{12}$ Therefore, it is possible to quantify reliably even the fine interstitial fibrosis as well as replacement scarring by our method.

Tissue sample selection and the direction in sectioning may also introduce bias. Because the distribution of fibrosis was not homogeneous, the extent of fibrosis in the transverse sections examined may not be representative of the entire slice. For this reason we also measured fibrosis in a second transverse section ( $1 \mathrm{~cm}$ below the first section) in five cases of hypertrophic cardiomyopathy. In all cases the extent of fibrosis was similar in the first and second sections examined. We also compared the percentage areas of fibrosis in transverse and longitudinal sections of the same tissue area in five cases of hypertrophic cardiomyopathy. The global and regional distribution of fibrosis in these sections was much the same $(r=0.91)$.

DATA ANALYSIS

We used one way analysis of variance with NeumanKeuls' multiple comparison test to compare regional myocardial fibrosis in each group and to compare global myocardial fibrosis among the three groups. We used paired $t$ test to compare myocardial fibrosis in the ventricular septum and left ventricular free wall in each group. Probability values of $p<0.05$ were regarded as statistically significant.

\section{Results}

Figure 2 shows the percentage area of fibrosis in the left ventricular wall-10.5 (4.3)\% (mean (SD)) in hypertrophic cardiomyopathy, $2.6(1.5) \%$ in hypertensive heart disease, and $1 \cdot 1(0.5) \%$ in normal hearts. The percentage area of fibrosis in those with hypertrophic cardiomyopathy was significantly greater than that in hypertensive heart disease or in normal hearts $(p<0.001)$. The percentage area of fibrosis was significantly greater in hypertensive heart disease than in normal hearts $(p<0.05)$.

\section{REGIONAL DISTRIBUTION OF FIBROSIS}

\section{Hypertrophic cardiomyopathy}

The percentage area of fibrosis was $13 \cdot 1(4 \cdot 8) \%$ in the ventricular septum and $7 \cdot 7(4.2) \%$ in the left ventricular free wall (Fig. 3) $(p<0.001)$. There was significantly more fibrosis in the ventricular septum $(p<0.001)$ and the left ventricular free wall $(p<0.01)$ in hypertrophic cardiomyopathy than in hypertensive heart disease. The percentage area of fibrosis was not significantly different in the right, middle, and the left thirds of the ventricular septum

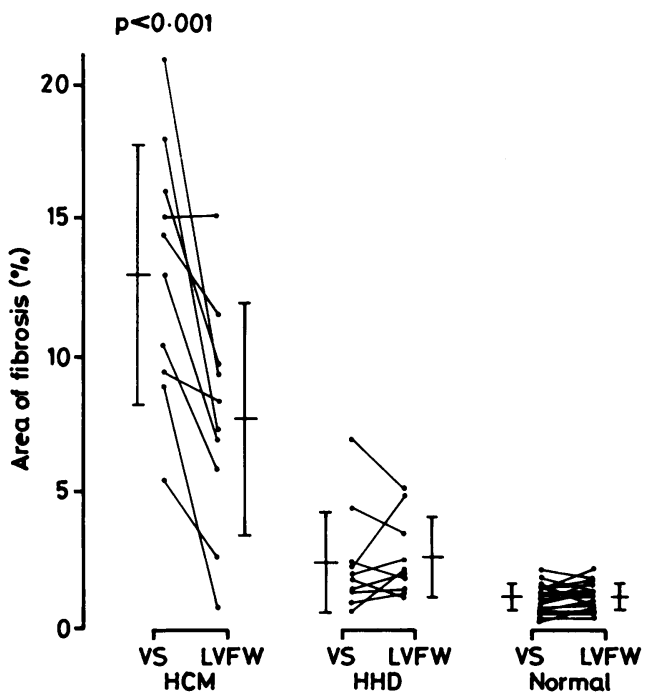

Fig. 3 Percentage area of fibrosis in ventricular septum (VS) and left ventricular free wall (LVFW) in hypertrophic cardiomyopathy (HCM), hypertensive heart disease (HHD), and normal controls. 

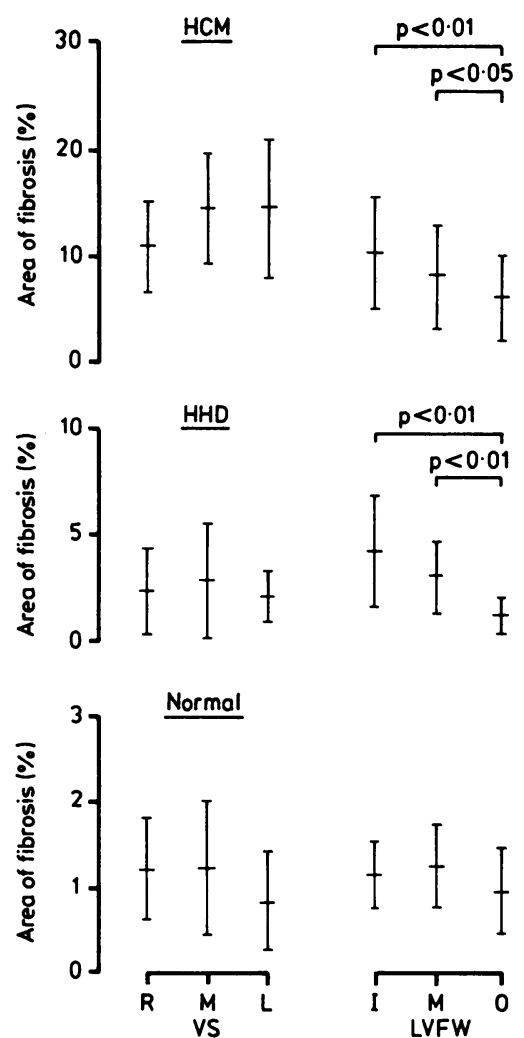

Fig. 4 Regional distribution of fibrosis in the left ventricular wall with hypertrophic cardiomyopathy (HCM), hypertensive heart disease (HHD), and normal controls. In HCM and HHD the percentage area of fibrosis increased from the outer to the inner third of the left ventricular free wall ( $L V F W)$. VS, ventricular septum; $R$, right; $M$, middle; $L$, left third of $V S$; I, inner; $M$, middle; $O$, outer third of LVFW.

$10 \cdot 7(4.4), 14.3(5 \cdot 2)$, and $14.4(6.7) \%$ respectively (Fig.4). In the left ventricular free wall, however, the percentage area of fibrosis increased significantly from the outer to the inner third of the wall $(5.8$ $(4.0) \%, 7.9(4.7) \%$, and $10.1(5.3) \%$ respectively; inner vs outer third, $\mathrm{p}<0.01$; middle vs outer third, $p<0.05)$. The percentage areas of fibrosis in the anterior, lateral, and posterior region of the left ventricular free wall $(7 \cdot 9(3 \cdot 7), 7 \cdot 4(6 \cdot 2)$, and $8 \cdot 1(6 \cdot 3) \%$ respectively) (Fig. 5) were not significantly different from one another. In each heart the distribution of fibrosis in these three regions of the left ventricular free wall varied, however.

\section{Hypertensive heart disease}

The percentage area of fibrosis was $2.4(1.9) \%$ in the ventricular septum and $2.6(1.5) \%$ in the left ventricular free wall (Fig. 3) (NS). The percentage areas of fibrosis were $2 \cdot 3(2 \cdot 0), 2 \cdot 8(2 \cdot 7)$, and $2 \cdot 0(1 \cdot 2) \%$ in the right, middle, and the left thirds of the ventricular septum respectively; and 4.1 (2.6), $2.9(1 \cdot 7)$, and $1.1(0.7) \%$ in the inner, middle, and outer thirds of the left ventricular free wall respectively (Fig.4). The differences in the percentage areas of fibrosis in the three regions of the ventricular septum were not significant, but the percentage area of fibrosis increased from the outer to the inner third of the left ventricular free wall (inner vs outer third and middle vs outer third, $p<0.01)$. The percentage areas of fibrosis were $2.3(1.1), 2.5(1.6)$, and $2.7(1.7) \%$ in the anterior, lateral, and posterior region of the left ventricular free wall respectively (Fig.5). There were no significant differences in percentage areas of fibrosis among these regions.

\section{Normal heart}

The percentage area of fibrosis was $1 \cdot 1(0 \cdot 5) \%$ in the ventricular septum and $1.1(0.5) \%$ in the left ventricular free wall (Fig. 3). The percentage area of fibrosis was $1.2(0.6), 1 \cdot 2(0.8)$, and $0.8(0.6) \%$ in the right, middle, and the left thirds of the ventricular septum respectively, and 1.1 (0.4), $1.2(0.5)$, and 0.9 $(0.5) \%$ in the inner, middle, and outer thirds of the left ventricular free wall respectively (Fig. 4). The percentage areas of fibrosis in the anterior, lateral, and posterior regions of the left ventricular free wall were $1 \cdot 1(0.6), 1 \cdot 1(0.5)$, and $1.2(0.6) \%$ respectively (Fig. 5). There were no significant differences in the intramural distribution of fibrosis in the left ventricular wall.

RELATION OF THE PERCENTAGE AREA OF FIBROSIS TO CLINICAL AND PATHOLOGICAL VARIABLES

We analysed the relation of the percentage areas of fibrosis to the septal thickness, septal:free wall ratio, heart weight, and the mode of death. There was no

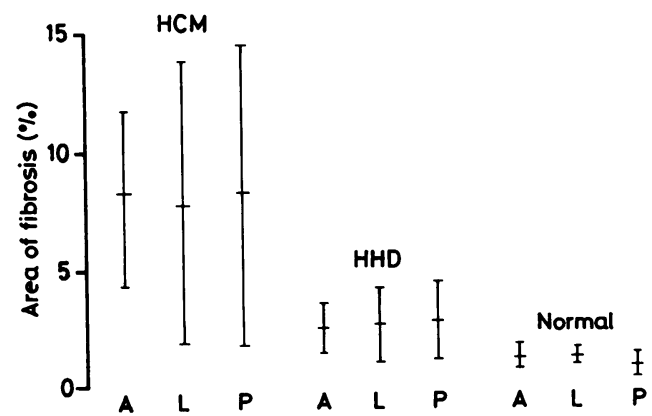

Fig. 5 Percentage area of fibrosis in anterior $(A)$, lateral $(L)$, and posterior $(P)$ wall of the left ventricular free wall in hypertrophic cardiomyopathy (HCM), hypertensive heart disease (HHD), and normal controls. These values are variable in each case but none of the regional differences is significant. 

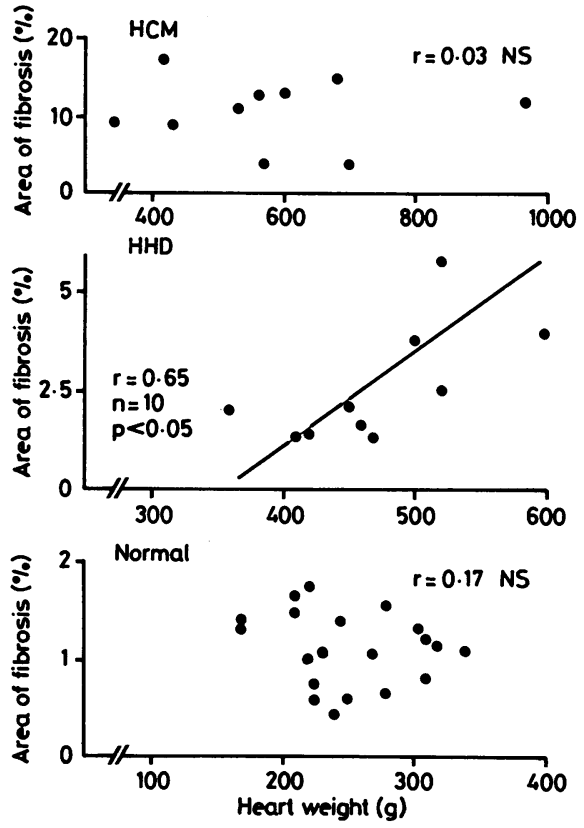

Fig. 6 Relations between the percentage area of fibrosis in the left ventricular wall and heart weight in hypertrophic cardiomyopathy (HCM), hypertensive heart disease (HHD), and normal controls.

correlation between the extent of fibrosis and septal thickness or the septal:free wall ratio in any heart.

In hypertrophic cardiomyopathy and in normal hearts there was no correlation between the extent of fibrosis and heart weight. In hypertensive heart disease, however, the extent of fibrosis correlated with heart weight $(r=0.65, p<0.05)$ (Fig. 6).

In hypertrophic cardiomyopathy sudden cardiac death was common in the cases of widespread fibrosis, but it was not seen in cases of slight fibrosis (Table) (Fig. 2).

\section{Discussion}

Our data show that in hearts with hypertrophic cardiomyopathy the extent of fibrosis was significantly greater than in hypertensive heart disease or in normal adult hearts, and that the percentage area of fibrosis was greater in the ventricular septum than in the left ventricular free wall.

Although the overall left ventricular systolic performance was generally in the normal range in hearts with hypertrophic cardiomyopathy, unit muscle performance was very low. ${ }^{13}$ Moreover, the ventricular septum is reported to be hypokinetic or adynamic in hypertrophic cardiomyopathy. ${ }^{14} 15$ These results suggest that, in addition to myocardial fibre disarray, ${ }^{5}$ the widespread fibrosis in hearts with hypertrophic cardiomyopathy is related to the impaired systolic unit muscle performance, particularly in the hypokinetic ventricular septum. Reduced compliance or increased stiffness of the left ventricle was more severe in hypertrophic cardiomyopathy than in hypertensive heart disease. ${ }^{16-18}$ Our results suggest that in addition to myocardial hypertrophy, increased fibrosis and myocardial fibre disarray ${ }^{19}$ also lead to the impaired diastolic compliance in hearts with hypertrophic cardiomyopathy.

Our data showed that in hearts with hypertrophic cardiomyopathy and hypertensive heart disease the percentage area of fibrosis increased from the outer to the inner third of the left ventricular free wall (transmural gradient of fibrosis). Analyses of the intramyocardial pressure, ${ }^{20-22}$ wall stress, ${ }^{23} 24$ and myocardial fibre diameter ${ }^{6}$ showed an increase from the epicardial to the endocardial layers of the left ventricular free wall, causing relative ischaemia on the endocardial layer. Moreover, the transmural gradient of fibrosis cannot be explained by myocardial fibre disarray because disarray is found predominantly in the middle and outer layers of the left ventricular free wall in hearts with hypertrophic cardiomyopathy ${ }^{5}$ and because myocardial fibre disarray is minimal in hypertensive heart disease. The transmural gradient of fibrosis in the left ventricular free wall with hypertrophic cardiomyopathy and hypertensive heart disease probably depends on the transmural gradient of tissue pressure, wall stress, and myocardial fibre diameter.

The extent of myocardial fibre disarray did not correlate with heart weight, septal thickness, or the septal:free wall ratio ${ }^{3-5}$ We found that the percentage area of fibrosis did not correlate with these variables in hypertrophic cardiomyopathy. But in hypertensive heart disease there was a correlation between the percentage area of fibrosis and heart weight, suggesting that myocardial hypertrophy caused by the increased wall stress due to systemic hypertension is most important in the pathogenesis of fibrosis in hypertensive heart disease. We believe that the wall stress was lower in hearts with hypertrophic cardiomyopathy than in hypertensive heart disease because no case of hypertrophic cardiomyopathy had hypertension. Therefore, increased wall stress cannot explain the significant increase in the percentage area of fibrosis in hearts with hypertrophic cardiomyopathy compared with hypertensive hearts.

Myocardial fibre disarray is typically but not exclusively found in hypertrophic cardiomyopathy. ${ }^{3-5}$ Widespread myocardial fibre disarray in the ventricular septum was evident in all our cases of hypertrophic cardiomyopathy but it was never seen 
in hypertensive heart disease or normal hearts. The percentage area of fibrosis was greater in the ventricular septum than in the left ventricular free wall in hearts with hypertrophic cardiomyopathy, but this was also true of the hypertensive and normal hearts. We have found that the percentage area of fibrosis in the tissue with disarray was greater than that in the tissue without disarray. ${ }^{25}$ This suggests that myocardial fibre disarray promotes fibrosis in hearts with hypertrophic cardiomyopathy. But we also found that even in the tissue without disarray the percentage area of fibrosis in hypertrophic cardiomyopathy was much greater than that of normal hearts or hypertensive hearts. ${ }^{25}$ Therefore, in addition to myocardial hypertrophy and fibre disarray, other unknown factors must be important in the pathogenesis of the widespread fibrosis in hearts with hypertrophic cardiomyopathy.

We thank Miss M Jinnai and Miss S Tomita for assistance with preparation of the paper. This project was supported in part by research grants from the Ministry of Health and Welfare of Japan and the Ministry of Education, Science and Culture of Japan.

\section{References}

1 Davies MJ. The cardiomyopathies. In: Pomerance A, Davies MJ, eds. Pathology of the heart. London: Blackwell Scientific Publications, 1975: 211-50.

2 Roberts WC, Ferrans VJ. Pathologic anatomy of the cardiomyopathies. Hum Pathol 1975; 6: 287-342.

3 Maron BJ, Roberts WC. Quantitative analysis of cardiac muscle cell disorganization in the ventricular septum of patients with hypertrophic cardiomyopathy. Circulation 1979; 59: 689-706.

4 Maron BJ, Anan TJ, Roberts WC. Quantitative analysis of the distribution of cardiac muscle cell disorganization in the left ventricular wall of patients with hypertrophic cardiomyopathy. Circulation 1981; 63: 882-94.

5 Fujiwara $H$, Hoshino T, Fujiwara T, Kawai C, Hamashima Y. Classification and distribution of myocardial fascicle and fiber disarray in 14 hearts with hypertrophic cardiomyopathy in $25 \mu$ thick sections. Ipn Circ I 1982; 46: 225-34.

6 Hoshino T, Fujiwara H, Kawai C, Hamashima Y. Myocardial fiber diameter and regional distribution in the ventricular wall of normal adult hearts, hypertensive hearts and hearts with hypertrophic cardiomyopathy. Circulation 1983; 67: 1109-16.

7 Fujiwara H, Hoshino T, Yamana K, et al. Number and size of myocytes and amount of interstitial space in the ventricular septum and in the left ventricular free wall in hypertrophic cardiomyopathy. Am $\mathcal{F}$ Cardiol 1983; 52: 818-23.

8 Sutton MGStJ, Lie JT, Anderson KR, O'Brien PC, Frye RL. Histopathological specificity of hypertrophic obstructive cardiomyopathy. Myocardial fibre disarray and myocardial fibrosis. Br Heart $\mathcal{F} 1980$; 44: 433-43.

9 Anderson KR, Sutton MGStJ, Lie JT. Histopathological types of cardiac fibrosis in myocardial disease. F Pathol 1979; 128: 79-85.

10 Baandrup U, Olsen EGJ. Critical analysis of endomyocardial biopsies from patients suspected of having cardiomyopathy I. Morphological and morphometric aspects. Br Heart $\mathcal{f} 1981$; 45: 475-86.

11 Matsubara O, Tanaka $M$, Kasuga T, Ishii T, Chino $M$. Idiopathic cardiomyopathy. The pathologic roles of arteriolopathy. Hum Pathol 1984; 15: 39-47.

12 Fujiwara $H$, Onodera $T$, Tanaka $M$, Fujiwara $T$, Kawai C, Hamashima Y. The reliability of the tracingmethod of fine cardiac fibrosis at a magnification of $\times 10$-Preliminary study for quantitative analysis of fibrosis in large tissue sections of hearts with cardiomyopathy. Heart and Vessels 1985; 1: 182-4.

13 Hirota Y, Furubayashi K, Kaku K, et al. Hypertrophic nonobstructive cardiomyopathy. A precise assessment of hemodynamic characteristics and clinical implications. Am $\mathcal{F}$ Cardiol 1982; 50: 990-7.

14 Cohen MV, Cooperman LB, Rosenblum R. Regional myocardial function in idiopathic hypertrophic subaortic stenosis. An echocardiographic study. Circulation 1975; 53: 842-7.

15 ten Cate FJ, Hugenholtz PG, Roelandt J. Ultrasound study of dynamic behaviour of left ventricle in genetic asymmetric septal hypertrophy. Br Heart $\mathcal{F} 1977$; 39: 627-33.

16 Gaasch WH, Battle WE, Oboler AA, Banas JS Jr, Levine HJ. Left ventricular stress and compliance in man. With special reference to normalized ventricular function curves. Circulation 1972; 45: 746-62.

17 Hirota Y. A clinical study of left ventricular relaxation. Circulation 1980; 62: 756-63.

18 Hirota Y. A clinical study of left ventricular diastolic properties. Fpn Circ $\mathcal{F}$ 1982; 46: 49-54.

19 Sanderson JE, Gibson DG, Brown DJ, Goodwin JF. Left ventricular filling in hypertrophic cardiomyopathy. An angiographic study. Br Heart $\mathcal{F} 1977$; 39: 661-70.

20 Baird RJ, Manktelow RT, Shah PA, Ameli FM. Intramyocardial pressure. A study of its regional variations and its relationship to intraventricular pressure. I Thorac Cardiovasc Surg 1970; 59: 810-23.

21 Armour JA, Randrall WC. Canine left ventricular intramyocardial pressures. Am $\mathcal{F}$ Physiol 1971; 220: 1833-9.

22 Stein PD, Marzilli M, Sabbah HN, Lee T. Systolic and diastolic pressure gradients within the left ventricular wall. Am F Physiol 1980; 238: H 625-30.

23 Wong AYK, Rautaharju PM. Stress distribution within the left ventricular wall approximated as a thick ellipsoidal shell. Am Heart f 1968; 75: 649-62.

24 Mirsky I. Left ventricular stresses in the intact human heart. Biophys f 1969; 9: 189-208.

25 Tanaka M, Onodera $T$, Der-Jinn Wu, Fujiwara $H$, Kawai C. The pathogenesis of myocardial fibrosis in hypertrophic cardiomyopathy-with special reference to myocardial fiber disarray [Abstract]. $\mathfrak{f p n}$ Circ $\mathfrak{f}$ (in press). 